\title{
Muscle Fiber and Motor Unit Behavior in the Longest Human Skeletal Muscle
}

\author{
A. John Harris, ${ }^{1}$ Marilyn J. Duxson, ${ }^{2}$ Jane E. Butler, ${ }^{3,4}$ Paul W. Hodges, ${ }^{3,4}$ Janet L. Taylor, ${ }^{3,4}$ and Simon C. Gandevia ${ }^{3,4}$ \\ Departments of ${ }^{1}$ Physiology and ${ }^{2}$ Anatomy, University of Otago, Dunedin 9001, New Zealand, ${ }^{3}$ Prince of Wales Medical Research Institute, Randwick, New \\ South Wales, Australia 2031, and ${ }^{4}$ University of New South Wales, Sydney, New South Wales, Australia 2052
}

The sartorius muscle is the longest muscle in the human body. It is strap-like, up to $600 \mathrm{~mm}$ in length, and contains five to seven neurovascular compartments, each with a neuromuscular endplate zone. Some of its fibers terminate intrafascicularly, whereas others may run the full length of the muscle. To assess the location and timing of activation within motor units of this long muscle, we recorded electromyographic potentials from multiple intramuscular electrodes along sartorius muscle during steady voluntary contraction and analyzed their activity with spike-triggered averaging from a needle electrode inserted near the proximal end of the muscle. Approximately $30 \%$ of sartorius motor units included muscle fibers that ran the full length of the muscle, conducting action potentials at $3.9 \pm 0.1$ $\mathrm{m} / \mathrm{s}$. Most motor units were innervated within a single muscle endplate zone that was not necessarily near the midpoint of the fiber. As a consequence, action potentials reached the distal end of a unit as late as $100 \mathrm{~ms}$ after initiation at an endplate zone. Thus, contractile activity is not synchronized along the length of single sartorius fibers. We postulate that lateral transmission of force from fiber to endomysium and a wide distribution of motor unit endplates along the muscle are critical for the efficient transmission of force from sarcomere to tendon and for the prevention of muscle injury caused by overextension of inactive regions of muscle fibers.

Key words: human muscle; motor unit; sartorius muscle; electromyography; muscle fiber; conduction velocity

\section{Introduction}

Most mammalian muscles, except in primates, comprise short muscle fibers that terminate intrafascicularly so that they are arranged in series. In cat hindlimb muscles, for example, most fibers are 22-30 mm long, and fascicles of muscle fibers are crossed regularly by bands of endplates (Loeb et al., 1987). In most human muscles, in contrast, fibers run tendon to tendon (Paul, 2001), and each fascicle is crossed by one band of endplates. A few muscles, however, including sartorius (Aquilonius et al., 1984; Heron and Richmond, 1993), gracilis (Paul, 2001), and latissimus dorsi (Snobl et al., 1998), have multiple endplate zones, which poses the questions of how their motor unit (MU) territories are organized and whether contractile activity is synchronized along their lengths. Length-tension properties of sarcomeres pose theoretical difficulties for muscle fiber stability and for the neural control of motor units (Gordon et al., 1966). If neighboring sarcomeres are activated, one with greater myofilament overlap might shorten, whereas its neighbor with less overlap would extend. Several physiological mechanisms, including force-velocity properties, ameliorate this problem (Morgan, 1985); however, they cannot overcome the problem raised when a single muscle fiber action potential propagates farther than $\sim 20 \mathrm{~mm}$ from its

Received March 8, 2005; revised June 29, 2005; accepted July 19, 2005.

This work was supported by grants from New Zealand Lottery Health Research (A.J.H., M.J.D.) and the National Health and Medical Research Council of Australia (S.C.G., J.E.B., P.W.H., J.L.T.).

Correspondence should be addressed to Dr. Simon Gandevia, Prince of Wales Medical Research Institute, Barker Street, Randwick, New South Wales, Australia 2031.E-mail: s.gandevia@unsw.edu.au.

DOI:10.1523/JNEUROSCI.0923-05.2005

Copyright $\odot 2005$ Society for Neuroscience $\quad$ 0270-6474/05/258528-06\$15.00/0 initiation. Then part of a fiber contracts, whereas other regions are relaxed and susceptible to damage from overextension.

Human sartorius muscle is $\sim 600 \mathrm{~mm}$ long in tall individuals and contains six to seven neurovascular compartments (Yang et al., 1998), each crossed by a band of endplates (Paul, 2001). It has mixed fiber types, including 50\% type I fibers (Johnson et al., 1973). The disaggregation of human sartorius muscle demonstrates that some muscle fibers terminate intrafascicularly, whereas others are $>200 \mathrm{~mm}$ long (Heron and Richmond, 1993). To investigate the organization and control of motor units in sartorius muscle, we recorded electromyographic potentials with intramuscular electrodes at multiple sites along sartorius muscle during steady voluntary contractions. Sartorius muscle is accessible and has a narrow cross section, making it possible to record from many of its units at any one level with a single electrode. A motor unit potential at one site was used as a trigger, and activity before and after each trigger was averaged at each other site. "Locked" potentials at intervals corresponding to a wave traveling at $\sim 4 \mathrm{~m} / \mathrm{s}$ (muscle fiber conduction velocity) (Andreassen and Arendt-Nielsen, 1987) would represent muscle fiber action potentials propagating to or from the trigger site. Locked potentials with latencies representing much faster apparent velocities would represent neurally linked units: either single motor units made up of muscle fibers with synapses in different endplate zones or, less likely, different units with firing synchronized by a common interneuron.

Our electrophysiological measurements showed that at least $30 \%$ of motor units included fibers with an average conduction velocity of $3.9 \pm 0.1 \mathrm{~m} / \mathrm{s}$ that crossed all recording sites and ran virtually the full length of the muscle. Thus, some human muscle 


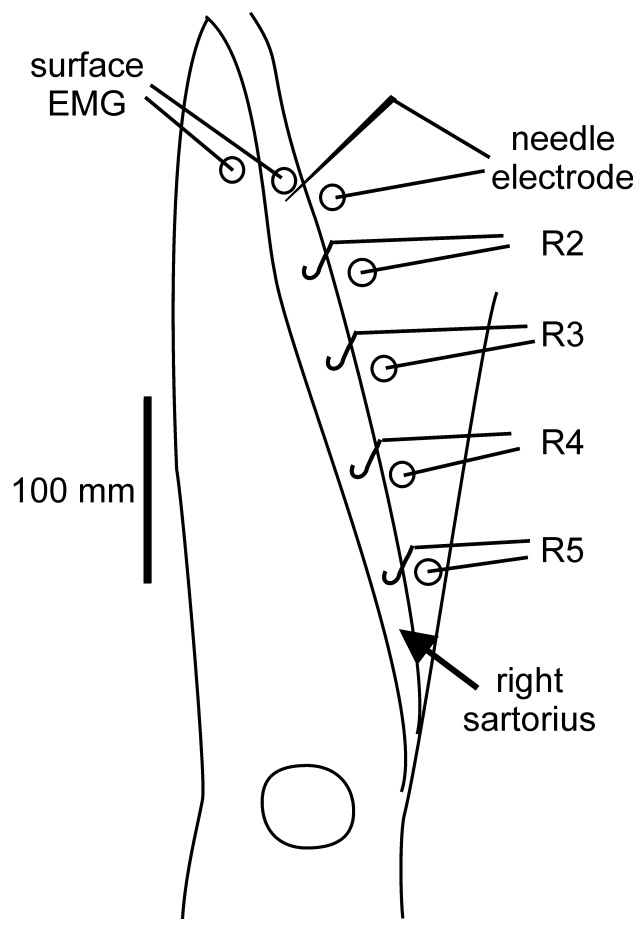

Figure 1. Arrangement of EMG recording electrodes (R) for recording from the human sartorius muscle. Additional details are given in Materials and Methods.

fibers behave as a single functional entity despite a length $>500$ $\mathrm{mm}$. Most units were activated from a single endplate band that was not necessarily near the center of the unit.

\section{Materials and Methods}

Electromyography subjects. Five male volunteers free of neurological or musculoskeletal disease were studied (see Table 1). Procedures were approved by the local ethics committee, and the studies were conducted according to the Declaration of Helsinki. The subjects reclined comfortably with their hips flexed to $120^{\circ}$ and knees flexed to $135^{\circ}$. The surface anatomy of their right sartorius muscle was defined by palpation combined with an ultrasound examination (Acuson XP 128/4; Siemens Medical Solutions, Mountain View, CA). In this position, their sartorius muscles ranged from 450 to $510 \mathrm{~mm}$ in length from origin to insertion.

Recordings. Electromyographic activity was recorded simultaneously from five sites along the muscle (Fig. 1). The most proximal site was $\sim 70$ $\mathrm{mm}$ (range, $50-90 \mathrm{~mm}$ ) from the origin of the muscle on the anterior superior iliac spine. Here a roving monopolar needle electrode was inserted and was moved within the cross section of the muscle to seek recording sites in which a single motor unit could be easily distinguished from other activity. Such single motor unit potentials were then used to trigger the averaging of activity recorded at the more distal recording sites. The most distal site was $\sim 70 \mathrm{~mm}$ from the axis of rotation of the knee, a point close to the initial insertion of the muscle. This site was chosen because it was proximal to where the cross-sectional area of the muscle diminishes as it nears its insertion and proximal to an overt intramuscular tendon, which was visualized by ultrasound. The three additional recording sites were equidistant between the proximal and most distal electrode sites.

Electromyographic activity was recorded at the four distal sites with hook-wire electrodes inserted perpendicular to the skin into the middle (thickest) part of the muscle via a 25 gauge needle under ultrasound guidance $(7 \mathrm{~cm}$ linear probe). Each electrode was made from insulated stainless-steel wire ( $75 \mu \mathrm{m}$ diameter) with $15 \mathrm{~mm}$ of insulation removed, including the "hook" of $5 \mathrm{~mm}$. This method was adopted to ensure that the wires could pick up crisp, multiunit activity from a large volume of the sartorius muscle. Each wire electrode was referred to a surface electrode (Ag-AgCl disk; $10 \mathrm{~mm}$ diameter) over the medial edge of the mus- cle at the same distance from the origin as the site of wire insertion. At the proximal site, an electromyogram (EMG) was recorded from a Tefloncoated monopolar electrode referenced to a surface electrode on the muscle just proximal to the needle insertion. Before needle insertion, the depth of the inner border of sartorius muscle was measured from the ultrasound image. This was the maximal depth for penetration of the needle electrode. A pair of surface electrodes was also placed transversely over the muscle close to the insertion of the monopolar electrode. The subject was grounded via a large flexible strap high on the upper thigh. The EMG signals were amplified and filtered (all intramuscular electrodes, $53 \mathrm{~Hz}$ to $3 \mathrm{kHz}$; proximal surface EMG recording, $53 \mathrm{~Hz}$ to 1.6 $\mathrm{kHz}$ ). All data were stored on disk with a CED 1401 interface (Cambridge Electronic Design, Cambridge, UK) for later analysis with Spike2 software. The sampling rates were $5 \mathrm{kHz}$ for the intramuscular EMG recordings and $2 \mathrm{kHz}$ for the proximal surface recording. In three preliminary studies, monopolar electrodes were inserted at two sites separated by 150-200 mm along the muscle, and simultaneous recordings showed propagation of some potentials during weak voluntary contractions. On this basis, the main study was designed with five recording sites widely spaced along the muscle. All recordings were monitored on-line on a multichannel oscilloscope as well as via the computer interface.

Recording protocol. The subject made a weak voluntary contraction while the needle electrode was being positioned to isolate a single motor unit at the proximal site. Subjects were then asked to keep the unit firing at a constant rate; unit activity was played on a loudspeaker to provide feedback. The single unit was used to generate trigger pulses with a dual time window discriminator (DDIS-1; Bak Electronics, Mt. Airy, MD). These pulses were used to generate spike-triggered averages of the surface EMG and the EMG recorded via the intramuscular electrodes. The signals were not rectified. Recordings were undertaken for 1.5-8 min. A new recording site was obtained by moving the monopolar electrode during a voluntary contraction. This procedure was repeated until at least 12 stable recording periods had been obtained. Motor unit potentials recorded via the surface electrodes and averaged on-line often gave a distinctive and consistent waveform for individual units; therefore, a preliminary check ensured that triggering unit potentials were not duplicated at different positions of the needle electrode. This was determined definitively from off-line analysis.

Analysis. In the first analysis, averages of all electromyographic signals were obtained for $100 \mathrm{~ms}$ before and after the trigger generated from the motor unit potential recorded at the proximal site. When necessary to verify particular responses, averages were repeated with the recording run broken down into segments. Only responses that could be identified consistently at the same latencies in all subaverages were accepted. To study additional motor units and to check on the results obtained with units triggered from the proximal needle electrode, the data were reanalyzed by making averages triggered from any single motor unit potentials with large signal-to-noise ratios identified in one of the more distal recordings. In the course of the analysis, most of the low-threshold units in which firing was evoked by voluntary contraction of the muscle were identified in several recording runs, and it was possible to display identical patterns of electromyographic activation with trigger potentials from several recording sites.

On the basis of the latency and distribution of events in the various recording electrodes, it was possible to determine the likely microanatomy of the triggering motor unit. To aid analysis, we modeled some possible arrangements and calculated the patterns of electromyographic potentials and their linkages to be expected from each model (see Fig. $2 A-D$ ). Potentials may reach the distal electrodes via a motor axon that branches (see Fig. $2 D$ ), muscle fiber conduction (see Fig. $2 A, B$ ), or a combination of both (see Fig. $2 C$ ). These models also illustrate how the analysis was performed. Records were arrayed in order from proximal to distal recording electrodes, with the distance between them proportional to their distance along the muscle. The slope of diagonal lines joining times of onsets of the dominant component of each potential gave the velocity of conduction. A uniform velocity of $\sim 4 \mathrm{~m} / \mathrm{s}$ corresponds to a volley of action potentials conducted along a group of muscle fibers (Stålberg, 1966; Nishizono et al., 1989). A substantially faster apparent velocity, $40-70 \mathrm{~m} / \mathrm{s}$, would correspond to records from muscle fibers 
activated by branches of an intramuscular nerve axon, as seen in the cat sartorius muscle (Loeb et al., 1987). In practice, the most common pattern was a potential that passed under two or more adjacent recording sites with a conduction velocity in the range of $3.4-4.5 \mathrm{~m} / \mathrm{s}$ and hence was compatible with conduction along muscle fibers (see Results). On the basis of the timing and direction of such propagation, it was possible to infer the likely longitudinal region of the muscle in which the relevant neuromuscular junctions were located and the distance over which propagation occurred. Given the limitations of sampling activity across the muscle at different levels when single centrally located electrodes were used, and signal-to-noise limits in the averaging, the present methods may underestimate the longitudinal distance over which muscle fibers from a motor unit projected.

\section{Results}

Our subjects were able to keep their trigger units firing at steady frequencies of $\approx 10 \mathrm{~Hz}$ for periods of $1.5-8 \mathrm{~min}$. We attempted to record from at least 12 different trigger units in each subject, but later off-line analysis showed that when searching for units at different depths and different penetration angles, we often picked up different components of the same motor unit. Thus, 42 MUs were identified in records from the needle electrode. Trigger units were unambiguously identified by the waveform of the averaged surface recording immediately adjacent to the needle electrode. On some occasions, potentials with a constant temporal relationship to trigger unit (i.e., locked potentials) were clearly seen at each recording electrode without any need for signal averaging (see Fig. 3), but usually they could only be revealed by averaging (see Fig. 4). As well as using potentials recorded by the needle electrode at the proximal end of the muscle as triggers, we also were able to discriminate off-line individual unit potentials in recordings from the wire electrodes positioned more distally and to use these as triggers in the analysis. An additional $22 \mathrm{MUs}$ were identified in this manner.

In total, the analyses distinguished 64 single motor units from five subjects. On 21 occasions, records from the intramuscular electrodes revealed potentials locked between all five electrodes; a total of 15 MUs were locked between four electrodes, 13 MUs were locked between three electrodes, and 11 MUs were locked between two electrodes. On four occasions, records from the intramuscular electrodes revealed no potentials linked to the trigger potential recorded from the needle electrode. A summary of the likely disposition of endplates for each unit and subject is shown in Figure 6; all locked potentials were consistent with models A-C, with a mean propagation velocity of $3.9 \pm 0.1 \mathrm{~m} / \mathrm{s}$ (Table 1). There were no short-latency linkages to indicate the existence of neural conduction to link subcomponents of motor units along the muscle (Fig. 2D).

Most of the MUs (54 of 64) had patterns of recorded potentials consistent with activation of muscle fibers at a single endplate either near the end of the muscle (Fig. $2 A$ ) or more central (Fig. 2B). Figures 3 and 4 show examples in which the main potential was propagated from a proximal region in the muscle to the most distal electrode. The behavior of a few motor units was consistent with that shown in Figure $2 C$, which illustrates the pattern of potentials anticipated if some muscle fibers in an MU were innervated at one endplate band but other fibers were innervated at a neighboring band. A critical feature of the model is that the shortest latency responses among the locked potentials should exhibit two discrete peaks separated by an interval in the

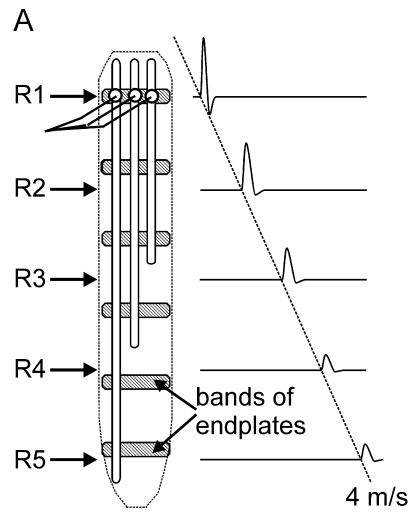

B
C

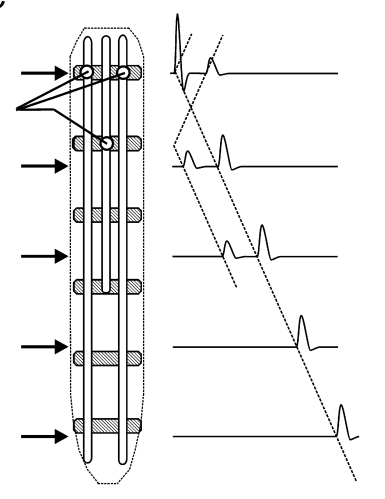

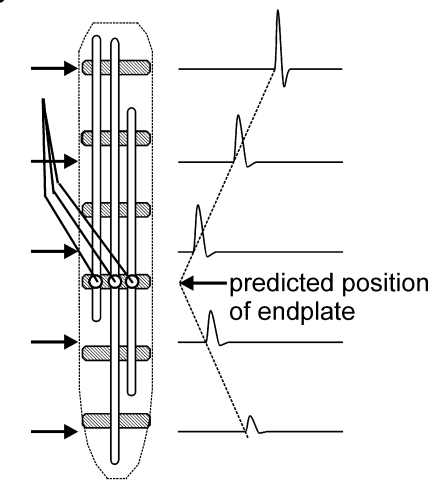

D

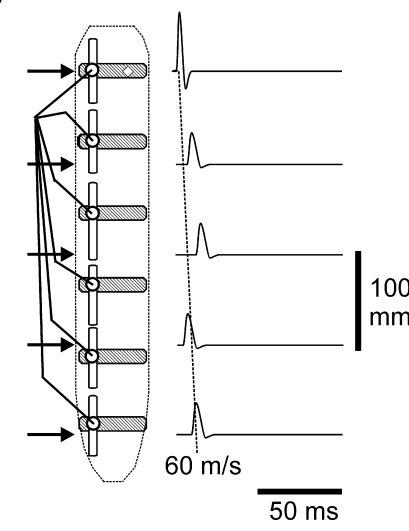

Figure 2. Theoretical patterns of EMG recordings from human sartorius muscle that are based on different models of motor unit microarchitecture. Each diagram represents a group of muscle fibers constituting one motor unit. The muscle is assumed to be $480 \mathrm{~mm}$ in length with six bands of endplates at uniform distances of $80 \mathrm{~mm}$. Muscle fiber action potentials are propagated at $4 \mathrm{~m} / \mathrm{s}$, and motor nerve action potentials are propagated at $60 \mathrm{~m} / \mathrm{s}$. Potentials recorded at the electrode $\mathrm{R} 1$ are used as the trigger to determine the time intervals of responses recorded at electrodes $R 2-R 5$. $A$, The motor unit receives all of its synaptic contacts in the most proximal band of endplates. Its muscle fibers penetrate varying distances along the muscle. In the graph at right, locked potentials at each recording site have latencies corresponding to conduction velocities of $4 \mathrm{~m} / \mathrm{s}$ (dotted line). $\boldsymbol{B}$, The motor unit receives all of its synaptic contacts at the fourth band of endplates. Locked potentials appear earlier than the proximal trigger potential. The graph of latency versus distance along the muscle consists of two straight lines corresponding to velocities of $4 \mathrm{~m} / \mathrm{s}$, intercepting at a point that marks the approximate position of the main innervation zone. $\boldsymbol{C}, A$ motor unit made up of fibers receiving synaptic contacts at one or the other of two adjacent endplate zones, resulting in "bi-peaked" electromyographic potentials. The interval between the two peaks is $20 \mathrm{~ms}$, corresponding to a conduction velocity of $4 \mathrm{~m} / \mathrm{s}$ if endplate zones are $80 \mathrm{~mm}$ apart. $D$, A motor unit made up of fibers in series. These fire slightly asynchronously because they are linked by a nerve fiber with a conduction velocity of 60 $\mathrm{m} / \mathrm{s}$ (dotted line). Other differences in the latencies of responses at each recording electrode are the result of muscle fiber conduction times that vary with the distances from each endplate to each recording electrode.

$10-20 \mathrm{~ms}$ range, corresponding to distances of $40-80 \mathrm{~mm}$ with a propagation velocity of $4 \mathrm{~m} / \mathrm{s}$. These two peaks should be recorded from at least two adjacent electrodes, and the predicted times of initiation of action potentials from each endplate in the 


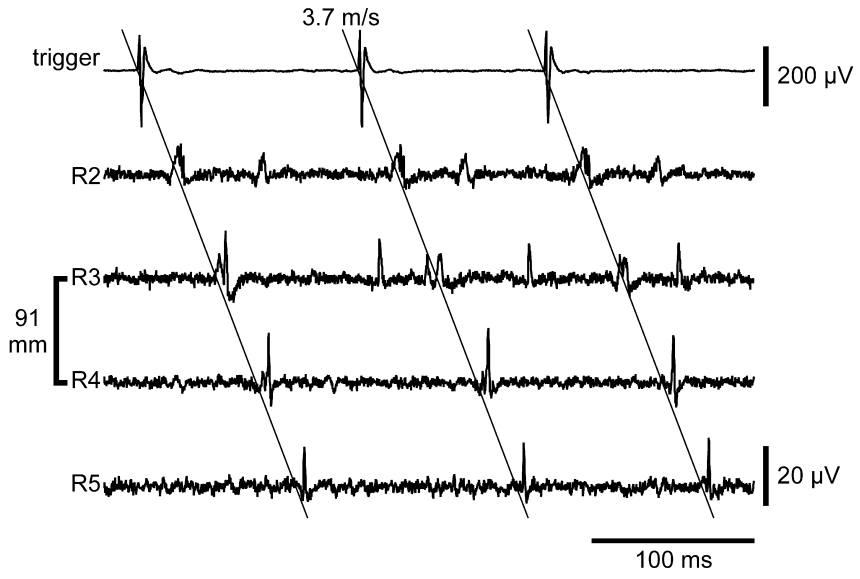

Figure 3. Locked potentials in raw EMG traces from five equidistant recording sites spaced at $91 \mathrm{~mm}$ intervals along a human sartorius muscle. The trigger unit fired three times and had a consistent potential shape. Action potentials propagate uniformly at $3.7 \mathrm{~m} / \mathrm{s}$. This value indicates that conduction is occurring along muscle fibers, extending the length of the muscle. It is not consistent with conduction via short muscle fibers activated by branches of a common motor axon. R, Recording electrode.

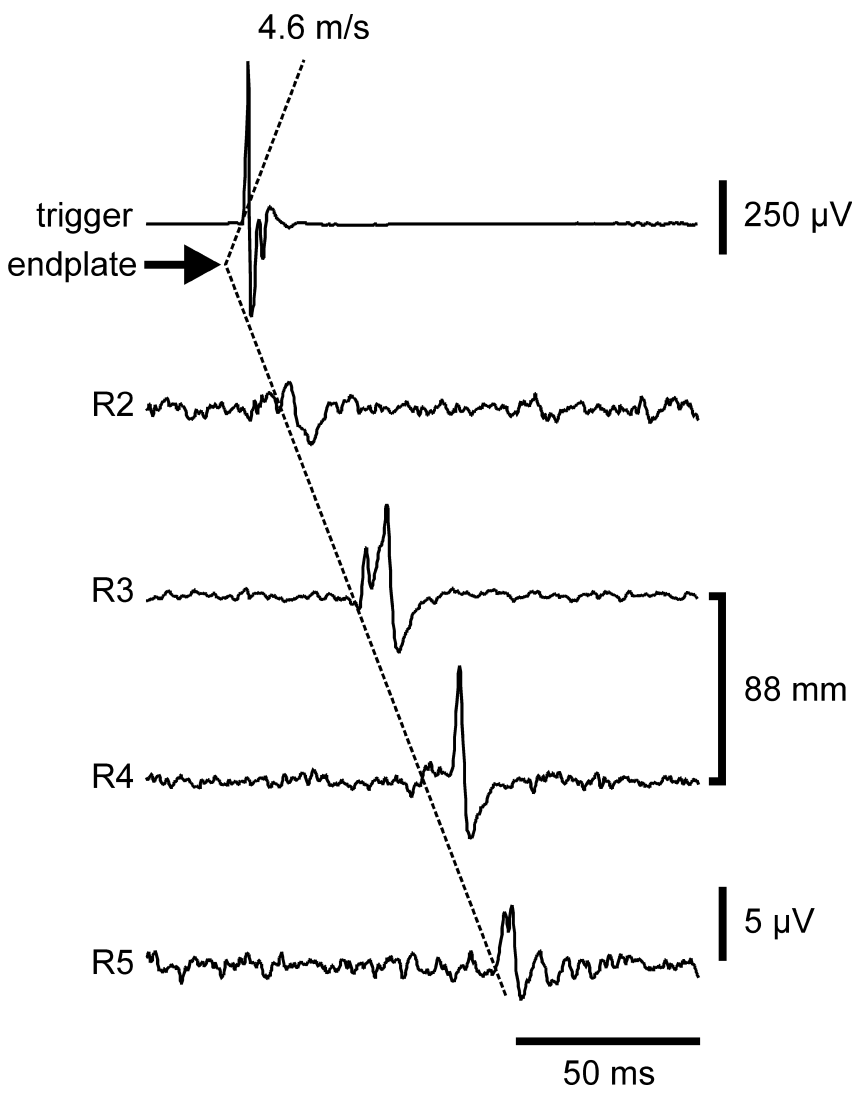

Figure 4. Locked potentials revealed by spike-triggered averaging from the timing of the single motor unit discharge to the four equidistant recording sites spaced at $88 \mathrm{~mm}$ intervals along the sartorius muscle. The averaged potentials propagated at $4.6 \mathrm{~m} / \mathrm{s}$ in the proximodistal direction with the initiating site between the site at which the triggering potential was recorded and recording electrode R2. Approximately 1500 sweeps were averaged. The propagation velocity and extent of conduction are consistent with conduction in muscle fibers along the length of the muscle.

unit should be the same. Four of the 64 MUs had responses that met this criterion: one was recorded at two adjacent electrodes, two were recorded at three adjacent electrodes, and one was recorded at four electrodes (Fig. 5). Intervals between peaks prop-

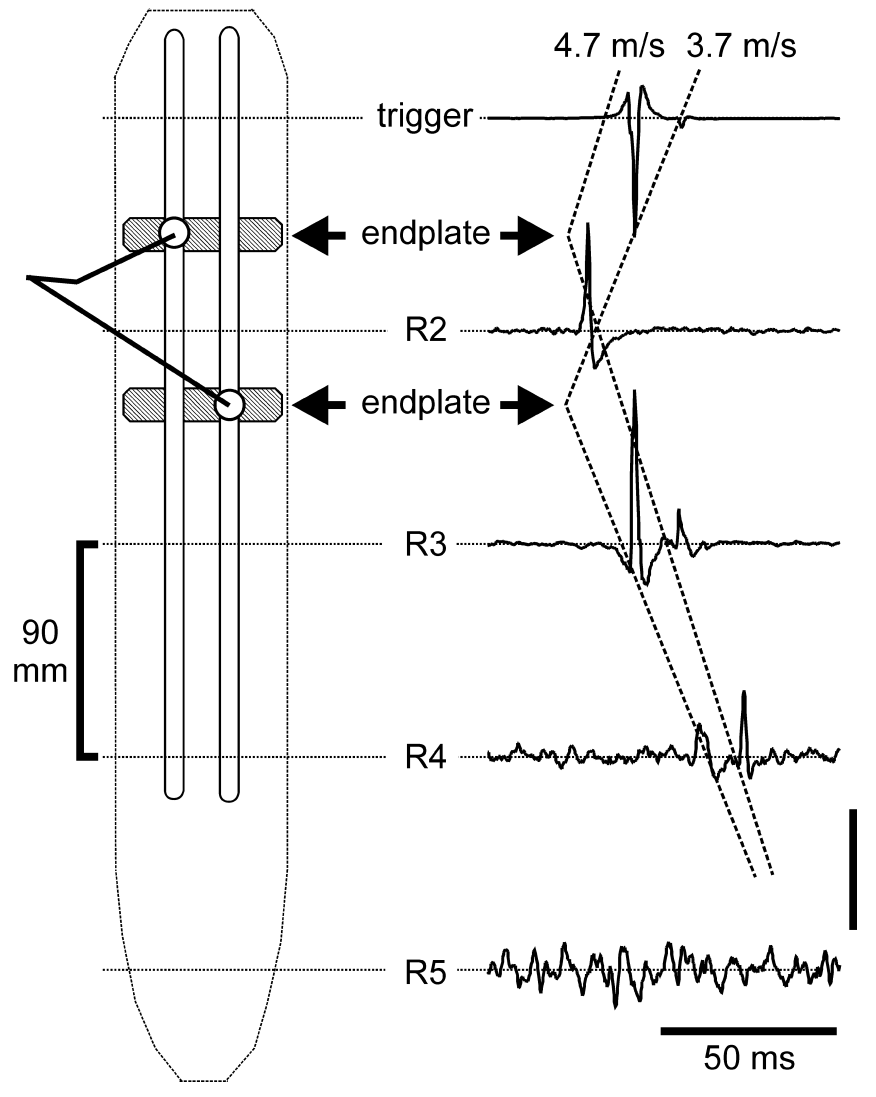

Figure 5. Example of a motor unit made up of muscle fibers probably innervated within two adjacent endplate bands. Recording electrodes were spaced at $90 \mathrm{~mm}$ intervals. On the left is a model diagram accounting for the pattern of potentials shown on the right (see also Fig. 2C). The double potentials recorded at electrodes R1, R3, and R4 may be accounted for if the unit received its innervation within two bands of endplates located $\sim 50 \mathrm{~mm}$ apart, and action potentials were initiated simultaneously in each band, $15 \mathrm{~ms}$ in advance of the trigger potential recorded at electrode R1. A total of 1800 sweeps were averaged. Calibration: trigger potential, $400 \mu \mathrm{V} ; \mathrm{R} 2, \mathrm{R} 3,50 \mu \mathrm{V} ; \mathrm{R} 4, \mathrm{R} 5,10 \mu \mathrm{V}$.

agating past one electrode ranged from 10 to $17 \mathrm{~ms}$, corresponding to distances between endplates of $40-68 \mathrm{~mm}$. Multiple innervation of single fibers by branches of a single motoneuron should give rise to responses similar to those in Figure $2 D$; these were not seen.

Nearly every unit occurred in more than one spike-triggered average as a result of using different members of the group of locked potentials as the trigger. This demonstrates the robust nature of the linkages. The appearance of the same unit in different analyses was recognized by several criteria, including the consistent waveforms recorded at particular electrodes, the waveform averaged from the proximal pair of surface electrodes, and the predicted position of the endplate.

\section{Discussion}

Theoretical arguments have been presented for long muscle fibers being less efficient than short fibers arranged in series. The basis for these arguments is that given the velocity of muscle action potentials $(\sim 4 \mathrm{~m} / \mathrm{s})$ and the general propensity of human motor units to discharge at $\sim 8-10 \mathrm{~Hz}$, sarcomere shortening cannot be well synchronized along the length of a long fiber (Loeb et al., 1987; Gans et al., 1989; Zenker et al., 1990). Nevertheless, many human muscles contain fibers $>100 \mathrm{~mm}$ long (Paul, 2001) that run the full length of each fascicle.

We investigated the functional microanatomy of motor units 
in human sartorius muscle because this is the most notable exception to the general rule that human muscles have single endplate zones (Paul, 2001). The sartorius muscle is the longest in the human body and has five to seven neurovascular compartments (Yang et al., 1998), each with a band of endplates traversing the muscle. Disaggregation experiments have shown that some fibers terminate intrafascicularly (Heron and Richmond, 1993), although results from a paper often quoted in textbooks, but based only on a superficial examination, suggest that others may run the full length of the muscle (Lockhart and Brandt, 1938). Our experiments show that at least $30 \%$ of motor units include a proportion of fibers running the full length of the muscle. The absence of electromyographic responses at the distal ends of the territories of some units may reflect limitations in the ability of the intramuscular electrodes to effectively sample unit activity, even after averaging (usually $>1000$ responses). This may be a particular problem in the units identified by triggering off-line from one of the more distal recording sites. Fifteen percent of units were recorded across the four intramuscular wire electrodes but not from the needle electrode. Thus, our figures probably underestimate the true proportion of the recorded motor units with fibers running the full length. Nonetheless, at least $55 \%$ of the units conducted $>340 \mathrm{~mm}$, and $31 \%$ conducted $>430 \mathrm{~mm}$. One limitation of our technique is that subjects made weak voluntary contractions so that only units of relatively low threshold were recruited. Thus, these proportions of long muscle fibers may not be representative of the entire muscle.

Sartorius and gracilis muscles are selectively spared in a number of human muscle diseases (Lamminen, 1990; Liu et al., 1993). This may reflect their possession of a proportion of muscle fibers that terminate intrafascicularly and hence are less susceptible to injury during eccentric contraction (Childers et al., 2002) than fibers that extend the full length of the muscle. Our findings indicate that in contrast to most human muscles in which all muscle fibers run end to end, $>40 \%$ of motor units in sartorius muscle contain fibers that are short compared with the length of the muscle fascicles.

Overall, our analysis revealed that endplate zones were distributed throughout the length of the muscle despite sampling being biased to the selection of motor units represented in the proximal part of the muscle. Of the 49 potentials that were recorded over three or more sites, the potential originated very close to the middle electrode (R3) in 5 MUs, more proximally in 28 MUs, and distally in 16 MUs (Fig. 5). The relative ease in recording locked potentials as far as $430 \mathrm{~mm}$ apart suggests that this technique will be of general use for sampling human muscle fiber conduction velocities and defining the distribution of components of single motor units within long muscles.
Our findings are relevant to the development of muscle. Fibers derived from secondary myotubes in guinea pig sternomastoid muscles terminate intrafascicularly, but fibers derived from primary myotubes run the full length of the muscle (Young et al., $2000)$. Cat limb muscles also include a small proportion of fibers $(\sim 1 \%)$ grouped into one or a few slow motor units that run full length and presumably also are derived from primary myotubes (Eldred et al., 1993). The incidence of units including full-length fibers in human sartorius muscle is too high to be accounted for in this way, and we interpret our data as showing that, in humans, fibers of secondary myotube origin can grow longitudinally to extend through most or all of the muscle. Figure 6 illustrates that $\sim 60 \%$ of motor units with fibers extending the full length of the muscle had endplates that were located toward one or the other end of the fibers rather than centrally, and this indicates that muscle fiber growth is not necessarily symmetrical about the endplate.

The ultimate function of sarcomere shortening is to exert force on muscle tendons to stabilize or move joints. Inactive regions of muscle fiber interpose an elastic compliance between 
active sarcomeres and myotendinous junctions. In human sartorius muscle, some motor units appear to be made up of muscle fibers that terminate intrafascicularly. A contraction of such a motor unit alone would result in the stretching of any inactive muscle fibers that were in series with the contracting fibers and could produce little overall tension. Other muscle fibers can be $>500 \mathrm{~mm}$ in length and reach from tendon to tendon; however, with endplates near one end of the muscle, sarcolemmal activation may take $>100 \mathrm{~ms}$ in a long fiber. This temporal delay is likely to mean that in a single twitch contraction initiated proximally, there will be a lag before tension is generated at the distal tendon, and during this time the less active regions of the fiber will be stretched. A corollary of the temporal dispersion of the contraction is that the twitch will produce much less force than if all sarcomeres could contract synchronously. The distortions created by these effects and the challenge to neural coordination must become more prominent in muscles with longer fascicles. Some force can be transmitted laterally between fibers (Street, 1983; Patel and Lieber, 1997; Huijing, 1999; Huijing and Baan, 2001; Monti et al., 2001). Lateral transmission of force occurs from z-lines to extracellular matrix via costameres and catenincadherin complexes (Young et al., 2000; Paul et al., 2002). Lateral adhesion plaques with dense accumulations of transverse collagen fibrils can be visualized in guinea pig muscles stained for acetylcholinesterase (Young et al., 2000). Similar dense spots of intramuscular acetylcholinesterase, not associated with endplates or myotendinous junctions, are prominent in human muscles (our unpublished observations). We suggest that there must be multiple pathways for force transmission from sarcomeres to muscle attachments.

If all motor unit endplates were at the same level in the muscle, lateral force transmission on its own would not be helpful. Then, at the onset of contraction, force production would still be limited by the compliance of nonactive regions of the muscle. This problem does not occur for sartorius muscle because the motor unit endplates are widely distributed along the muscle. Our findings show that this wide distribution holds true within a group of low-threshold motor units (Fig. 6). Presumably, this ensures that at least some parts of muscle fibers would be contracting all along the muscle whenever several motoneurons are activated together. The longitudinally distributed initiation of contraction, combined with lateral transmission of force, should increase the rate of force production and protect inactive sarcomeres from overextension and damage.

\section{References}

Andreassen S, Arendt-Nielsen L (1987) Muscle fiber conduction velocity in motor units of the human anterior tibial muscle: a new size principle parameter. J Physiol (Lond) 391:561-571.

Aquilonius SM, Askmark H, Gillberg PG, Nandedkar S, Olsson Y, Stålberg E (1984) Topographical localization of motor endplates in cryosections of whole human muscles. Muscle Nerve 7:287-293.

Childers MK, Okamura CS, Bogan DJ, Bogan JR, Petroski GF, McDonald K, Kornegay JN (2002) Eccentric contraction injury in dystrophic canine muscle. Arch Phys Med Rehabil 83:1572-1578.

Eldred E, Ounjian M, Roy RR, Edgerton VR (1993) Tapering of the intrafas- cicular endings of muscle fibers and its implications to relay of force. Anat Rec 236:390-398.

Gans C, Loeb GE, De Vree F (1989) Architecture and consequent physiological properties of the semitendinosis muscle in domestic goats. J Morphol 199:287-297.

Gordon AM, Huxley AF, Julian FJ (1966) The variation in isometric tension with sarcomere length in vertebrate muscle fibres. J Physiol (Lond) 184:170-192.

Heron MI, Richmond FJ (1993) In-series fiber architecture in long human muscles. J Morphol 216:35-45.

Huijing P (1999) Muscular force transmission: a unified, dual or multiple system? A review and some explorative experimental results. Arch Physiol Biochem 107:292-311.

Huijing PA, Baan GC (2001) Myofascial force transmission causes interaction between adjacent muscles and connective tissue: effects of blunt dissection and compartmental fasciotomy on length force characteristics of rat extensor digitorum longus muscle. Arch Physiol Biochem 109:97-109.

Johnson MA, Polgar J, Weightman D, Appleton D (1973) Data on the distribution of fiber types in thirty-six human muscles. An autopsy study. J Neurol Sci 18:111-129.

Lamminen AE (1990) Magnetic resonance imaging of primary skeletal muscle diseases: patterns of distribution and severity of involvement. $\mathrm{Br} \mathrm{J}$ Radiol 63:946-950.

Liu GC, Jong YJ, Chiang CH, Jaw TS (1993) Duchenne muscular dystrophy: MR grading system with functional correlation. Radiology 186:475-480.

Lockhart RD, Brandt W (1938) Length of striated muscle fibres. J Anat $72: 470$.

Loeb GE, Pratt CA, Chanaud CM, Richmond FJ (1987) Distribution and innervation of short, interdigitated muscle fibers in parallel-fibered muscles of the cat hindlimb. J Morphol 191:1-15.

Monti RJ, Roy RR, Edgerton VR (2001) Role of motor unit structure in defining function. Muscle Nerve 24:848-866.

Morgan DL (1985) From sarcomeres to whole muscles. J Exp Biol 115:69-78.

Nishizono H, Kurata H, Miyashita M (1989) Muscle fiber conduction velocity related to stimulation rate. Electroencephalogr Clin Neurophysiol 72:529-534.

Patel TJ, Lieber RL (1997) Force transmission in skeletal muscle: from actomyosin to external tendons. Exerc Sport Sci Rev 25:321-363.

Paul AC (2001) Muscle length affects the architecture and pattern of innervation differently in leg muscles of mouse, guinea pig, and rabbit compared to those of human and monkey muscles. Anat Rec 262:301-309.

Paul AC, Sheard PW, Kaufman SJ, Duxson MJ (2002) Localization of alpha 7 integrins and dystrophin suggests potential for both lateral and longitudinal transmission of tension in large mammalian muscles. Cell Tissue Res 308:255-265.

Snobl D, Binaghi LE, Zenker W (1998) Microarchitecture and innervation of the human latissimus dorsi muscle. J Reconstr Microsurg 14:171-177.

Stålberg E (1966) Propagation velocity in human muscle fibres in situ. Acta Physiol Scand 70:1-112.

Street SF (1983) Lateral transmission of tension in frog myofibres: a myofibrillar network and transverse cytoskeletal connections are possible transmitters. J Cell Physiol 114:346-364.

Yang D, Morris SF, Sigurdson L (1998) The sartorius muscle: anatomic considerations for reconstructive surgeons. Surg Radiol Anat 20:307-310.

Young M, Paul A, Rodda J, Duxson M, Sheard P (2000) Examination of intrafascicular muscle fiber terminations: implications for tension delivery in series-fibered muscles. J Morphol 245:130-145.

Zenker W, Snobl D, Boetschi R (1990) Multifocal innervation and muscle length. A morphological study on the role of myo-myonal junctions, fiber branching and multiple innervation in muscles of different size and shape. Anat Embryol 182:273-283. 